\title{
Diminishing Use of Liver Biopsy among Liver Transplant Recipients for Hepatitis C
}

\author{
Elizabeth Aby ${ }^{1}$, Melissa A. Jimenez ${ }^{2}$, Jonathan F. Grotts ${ }^{1}$, Vatche Agopian ${ }^{2}$, \\ Samuel W. French ${ }^{3}$, Ronald W. Busuttil ${ }^{2}$ and Sammy Saab*1,2 \\ ${ }^{1}$ Departments of Medicine at the University of California at Los Angeles, Los Angeles, California, USA; ${ }^{2}$ Departments of \\ Surgery at the University of California at Los Angeles, Los Angeles, California, USA; ${ }^{3}$ Departments of Pathology at the
} University of California at Los Angeles, Los Angeles, California, USA

\begin{abstract}
Background and Aims: Hepatitis C virus (HCV) cirrhosis is the leading indication for liver transplantation in the United States and recurrent HCV following liver transplantation is a major cause of allograft loss and mortality. Liver biopsies are commonly used to identify recurrent HCV and determine the need for antiviral therapy. The introduction of directacting antiviral agents (DAAs) has changed the management of recurrent HCV infection. This study aimed to describe the role of liver biopsies in liver transplant recipients with HCV after the introduction of DAAs. Methods: A retrospective analysis was performed looking at the rate of liver biopsies post-liver transplantation for HCV. The analysis included 475 adult liver transplants for hepatitis $C$ performed at the University of California, Los Angeles from January 1, 2006 to October 1, 2015. Patients were divided into two eras, pre- and post-introduction of DAAs on December 1, 2013. Results: In the era before the introduction of DAAs, the percentage of patients biopsied was significantly higher compared to the era after the introduction of DAAs $(56.1 \%$ vs. $26.9 \%$, $p<0.001)$. Conclusion: The introduction of DAAs has changed the management of liver biopsy following liver transplantation and the management of recurrent HCV. Given that DAAs are well tolerated and have high efficacy, liver biopsies are no longer routinely used to justify the use antiviral therapy following liver transplantation.
\end{abstract}

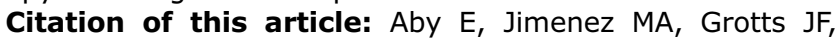
Agopian V, French SW, Busuttil RW, et al. Diminishing use of liver biopsy among liver transplant recipients for hepatitis C. J Clin Transl Hepatol 2017;5(3):197-202. doi: 10.14218/ JCTH.2016.00073.

\section{Introduction}

Hepatitis C virus (HCV) affects over 3 million individuals in the United States. ${ }^{1}$ Patients with HCV, however, are often unaware of their disease because the majority of acutely infected patients are asymptomatic. ${ }^{1} \mathrm{HCV}$ is an important medical concern for our society, as the rate of death from HCV infections is surpassing the rate of death from human immunodeficiency virus infections in the United States. ${ }^{2}$ $\mathrm{HCV}$ is currently the leading indication for liver transplantation in the United States, ${ }^{3}$ while recurrent HCV post-liver transplantation is universal and a major cause of allograft loss and mortality. ${ }^{4}$ Liver transplantation into an HCV infected recipient leads to $\mathrm{HCV}$-induced allograft hepatitis in $80 \%$ of recipients at 5 years. ${ }^{5}$

Historically, the diagnosis and management of $\mathrm{HCV}$ pre- and post-liver transplantation has relied on liver biopsy. Indications for liver biopsy post-transplant in the past have included evaluating the disease stage and grade, monitoring response to therapy, and diagnosis of disease. Liver biopsy, however, is invasive and associated with bleeding, pain and infection. A retrospective analysis of percutaneous and transjugular liver biopsies for orthotropic liver transplant patients in 286 patients showed 3.0\% overall incidence of liver biopsy related infection. ${ }^{6}$

Liver biopsies are also subject to sampling error. One study examined chronic HCV patients who received multiple concurrent liver biopsies and found a difference of at least one histologic stage in approximately one-third of patients, suggesting that biopsies do not always provide definitive results. ${ }^{7}$ Given the risks of and shortcomings associated with liver biopsy, new modalities have been developed for the diagnosis and management of chronic hepatitis and fibrosis, including serological tests and imaging studies. Advances in non-invasive imaging and serologic techniques for the assessment of fibrosis, as well as the introduction of new direct-acting antiviral agents (DAAs) for $\mathrm{HCV}$, have changed the indications for liver biopsy.

Strategies for the treatment of HCV have evolved since the use of subcutaneous injections of alpha interferons (IFN- $\alpha$ ) in 1986. In 1988, ribavirin was combined with IFN- $\alpha$ for improved, sustained virologic response (SVR). ${ }^{8}$ The first DAAs introduced in 2011 were protease inhibitors, telaprevir and boceprevir, although these agents required the concomitant use of interferon and ribavirin due to viral resistance associated with monotherapy. ${ }^{9}$ The approval of additional DAAs in the fall of 2013, including sofosbuvir and simeprevir, 
as part of interferon-free regimens has changed the landscape of HCV treatment. The removal of interferon increases the number of patients who may be able to tolerate treatment for HCV given the reduced side effects associated with treatment. Furthermore, DAAs are effective. DAAs have been shown to have SVR of greater than $90 \%$, shorter and more tolerable treatment regimens, and high barriers to resistance. ${ }^{10}$ Of note, there is a decline in SVR in patients with $\mathrm{HCV}$ cirrhosis treated with sofosbuvir and simeprevir that correlates with worsening Child-Pugh score; Child-Pugh cirrhosis classes A, B and C were associated with an SVR of $87 \%, 77 \%$ and $67 \%$ respectively. ${ }^{11}$

The aim of this study was to determine how the practice of liver biopsy is changing given the introduction of DAAs for the treatment of HCV. The hypothesis of our study was that over the last 10 years there has been a decreased incidence of liver biopsies after transplantation for $\mathrm{HCV}$, especially after the introduction of DAAs.

\section{Methods}

A single-center, retrospective analysis was conducted, including all liver biopsies performed at the University of California, Los Angeles (UCLA) Medical Center between January 1, 2006 and October 1, 2015 after liver transplantation for HCV.

Exclusion criteria included patients under the age of 18 at the time of transplant, liver biopsies performed within 30 days of transplant, liver biopsies performed to biopsy a hepatic mass, patients who died within 12 months of transplantation, and biopsies after a second transplant for liver failure (Fig. 1). Patients were excluded if they died within
12 months of transplantation, as they would not have an adequate chance for biopsy post-transplantation. Patients with liver biopsies after repeat liver transplantation were excluded, as they were transplanted due to allograft failure.

The timeframe of observation was divided into two discrete periods: "Era 1" defines the time period from January 1 , 2006 to November 30, 2013; and "Era 2" includes the time period from December 1, 2013 until October 1, 2015. Era 2 was defined by the Federal Drug Administration's approval of DAAs as part of interferon-free regimens. At UCLA, there has never been a protocol in place for liver biopsies in HCVrelated liver transplant patients before or after the introduction of DAAs.

All patients had at least 6 months of follow-up. Data were obtained by review of the medical charts and the UCLA Liver Transplant database. Demographic data (age, sex, race/ ethnicity, and body mass index (BMI)), HCV genotype, liver pathology results and laboratory data were recorded.

Continuous variables are presented as medians with interquartile range, and discrete data are presented as number of group with percent of group. A Wilcoxon rank sum test was used to compare continuous data between eras and a Fisher's exact or chi-square test was used for discrete data. A Cox proportional hazards model was fit to analyze time to first biopsy stratified by era. All tests were two-sided, with a $p$-value below 0.05 indicating statistical significance. The $\mathrm{R}$ statistical programming environment was used for all data analysis (R Core Team; Vienna, Austria).

The study was approved by the UCLA Institutional Review Board (IRB Study Number: 16-000091).

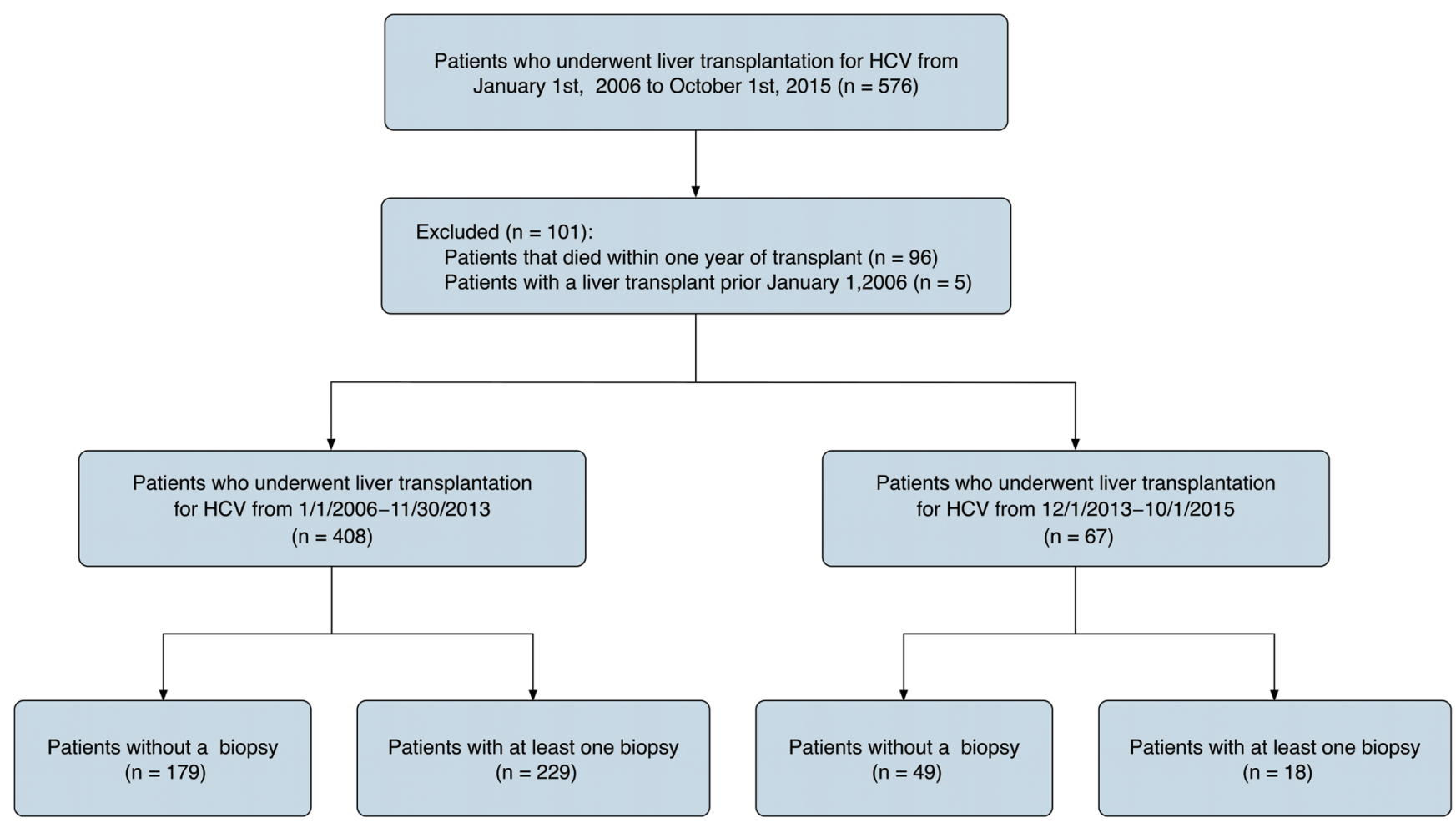

Fig. 1. Flow chart of patients who met inclusion and exclusion criteria for the study population. 
Aby E. et al: Role of liver biopsy for HCV post-LT

\section{Results}

A total of 576 liver transplants were performed at UCLA from January 1, 2006 to October 1, 2015 for hepatitis C. A total of 475 patients were included in our analysis. Ninety-six patients were excluded because they died within 1 year of transplant and five were excluded because they had undergone their first liver transplant prior to study initiation (Fig. 1). Table 1 shows baseline characteristics of the overall cohort, stratified by era. A similar proportion of patients were male in each era $(70.1 \%$ in Era 1 vs. $65.7 \%$ in Era 2, $p=0.48$ ). The median age (in years) at the time of liver transplant was 57.8 in Era 1 compared to 60.1 in Era $2(p=0.055)$. There were no statistically significant differences between eras in the percentage of patients who had concomitant hepatitis $B$ virus infection, non-alcoholic steatohepatitis (NASH), alcoholic liver disease, or hepatocellular carcinoma.

There were no statistically significant differences between eras in lab values, including international normalized ratio (INR), total bilirubin, serum creatinine (SCr), platelets, alanine aminotransferase (ALT), or aspartate aminotransferase (AST) at the time of transplant (Table 2). No differences in HCV viral load at the time of transplant were noted between eras (Table 2). The hepatitis $C$ genotype is not known for half of the patients in Era 1, whereas the genotype is not known in a quarter of patients in Era 2. The most common hepatitis C genotype for patients in both eras is genotype 1 .

The number of biopsies performed per year divided by the number of liver transplant patients remained relatively steady at $0.25-0.35$ from 2006 to 2013, with a decline noted after 2013 (Fig. 2). There was an increase in the number of patients biopsied as a proportion of liver transplant patients in 2007 compared to 2006, 0.28 compared to 0.52 . The proportion returned to 2006 levels in the following year and remained consistent until 2013, ranging from 0.27 to 0.35 . The proportion dropped to below 0.15 after 2013 .

In the era before the introduction of DAAs, the percentage of patients biopsied was significantly higher compared to the era after the introduction of DAAs (Table $1 ; 56.1 \%$ vs. $26.9 \%, p<0.001)$. The percentage of patients biopsied at least once within 12 months of liver transplant was $46.4 \%$ in the era prior to the introduction of DAAs compared to

Table 1. Baseline characteristics for patients transplanted for HCV separated by era, January 1, 2006 to 0 ctober 1, 2015 ( $n=475)$

\begin{tabular}{|c|c|c|c|c|c|}
\hline Variables & Era 1 & $n$ & Era 2 & $n$ & $p$-Value \\
\hline Age at transplant ${ }^{*}$ & $57.8(20.4-75.3)$ & 408 & $60.1(28-75.8)$ & 67 & 0.055 \\
\hline Sex & & & & & 0.476 \\
\hline Female & $29.9 \%$ & 122 & $34.3 \%$ & 23 & \\
\hline Male & $70.1 \%$ & 286 & $65.7 \%$ & 44 & \\
\hline \multicolumn{6}{|l|}{ Race } \\
\hline White & $34.3 \%$ & 140 & $35.8 \%$ & 24 & \\
\hline Asian & $7.8 \%$ & 32 & $1.5 \%$ & 1 & \\
\hline Black & $6.6 \%$ & 27 & $9.0 \%$ & 6 & \\
\hline Hispanic & $30.4 \%$ & 124 & $49.3 \%$ & 33 & \\
\hline Other & $20.8 \%$ & 85 & $4.5 \%$ & 3 & \\
\hline BMI* $^{*}$ & $25.9(15.3-46.8)$ & 408 & $27.5(19.9-47.5)$ & 67 & 0.055 \\
\hline Hepatitis B Liver disease & & & & & 0.488 \\
\hline No & $96.1 \%$ & 392 & $98.5 \%$ & 66 & \\
\hline Yes & $3.9 \%$ & 16 & $1.5 \%$ & 1 & \\
\hline Alcoholic Liver Disease & & & & & 0.797 \\
\hline No & $93.1 \%$ & 380 & $92.5 \%$ & 62 & \\
\hline Yes & $6.9 \%$ & 28 & $7.5 \%$ & 5 & \\
\hline NASH & & & & & 0.367 \\
\hline No & $98.3 \%$ & 406 & $98.5 \%$ & 66 & \\
\hline Yes & $0.5 \%$ & 2 & $1.5 \%$ & 1 & \\
\hline Hepatocellular carcinoma & & & & & 0.293 \\
\hline No & $49.0 \%$ & 200 & $41.8 \%$ & 28 & \\
\hline Yes & $51.0 \%$ & 208 & $58.2 \%$ & 39 & \\
\hline Patients biopsied & & & & & $<0.001$ \\
\hline No & $43.9 \%$ & 179 & $73.1 \%$ & 49 & \\
\hline Yes & $56.1 \%$ & 229 & $26.9 \%$ & 18 & \\
\hline Days to first biopsy* & $150(32-3102)$ & & $67.5(35-504)$ & & 0.001 \\
\hline
\end{tabular}

*Continuous variables presented as median (range).

Abbreviations: BMI, body mass index; NASH, Non-alcoholic steatohepatitis. 
Table 2. Laboratory values at the time of liver transplant for patients transplanted for HCV separated by era, January 1, 2006 to October 1, $2015(n=475)$

\begin{tabular}{llll}
\hline & Era 1 & Era 2 & $p$-Value \\
\hline $\begin{array}{lll}\text { Genotype } \\
1\end{array}$ & $146(35.8 \%)$ & $40(59.7 \%)$ & \\
2 & $16(3.9 \%)$ & $5(7.5 \%)$ & \\
3 & $18(4.4 \%)$ & $5(7.5 \%)$ & \\
4 & $13(3.2 \%)$ & $1(1.5 \%)$ & \\
6 & $5(1.2 \%)$ & $0(0 \%)$ & \\
$\quad$ Unknown & $210(48.5 \%)$ & $16(23.9 \%)$ & \\
INR $^{*}$ & $1.5(1-4)$ & $1.6(1-3.7)$ & 0.559 \\
Total & 3.9 & 2.7 & 0.734 \\
Bilirubin $^{*}$ & $(0.3-83.4)$ & $(0.3-59.1)$ & \\
SCR $^{*}$ & $1.1(0.2-10.1)$ & $1.1(0.3-3.3)$ & 0.259 \\
Platelets $^{*}$ & $61(10-319)$ & $55(13-272)$ & 0.181 \\
AST $^{*}$ & $79(18-12278)$ & $93(20-3017)$ & 0.119 \\
ALT $^{*}$ & $53(4-7279)$ & $66(13-1148)$ & 0.227 \\
HCV RNA $^{*}$ & 513000 & 206500 & 0.299 \\
& $(25-6.9 e+08)$ & $(43-6.9 \mathrm{e}+07)$ & \\
\hline
\end{tabular}

* Continuous variables presented as median (range).

Abbreviations: ALT, alanine aminotransferase; AST, aspartate aminotransferase INR, international normalized ratio; HCV RNA, hepatitis C virus RNA; SCr, serum creatinine.

$25.9 \%$ of patients after the introduction of DAAs (Table 3 ). When comparing the timelines to first biopsy, rates of biopsy appear similar over the first 100 days post-transplant between patients in each era (Fig. 3). However, after 100 days, patients in Era 2 received fewer biopsies and this trend persisted to 365 days post-transplant.

In Era 1, the indication for liver biopsy within the first year was abnormal liver tests in $89.9 \%$ of patients, HCV staging in $8.9 \%$ of patients and 'other' in $1.2 \%$ of patients. In Era 2, the indication for liver biopsy within the first year was abnormal liver tests in all patients; there were no biopsies performed to determine HCV staging.

\section{Discussion}

The introduction of DAAs is changing the management of HCV liver disease pre-and post-liver transplantation and consequently is impacting the use of liver biopsies. We reviewed the rate of liver biopsies before and after the introduction of DAAs and found that post-DAA introduction, the percentage of patients receiving liver biopsies post-transplantation decreased 2-fold.

Recurrence of HCV post-transplantation is nearly universal and accounts for over $65 \%$ of allograft failures and deaths. ${ }^{4}$ Eradication of HCV prior to liver transplant is often preferred to treatment post-transplant, as it significantly reduces the post-transplant recurrence risk and potentially stabilizes or improves liver disease. However, the response rates to DAAs in patients with cirrhosis are reduced and patients with cirrhosis often require a longer duration of therapy, which substantially increases cost per cure.

Given that HCV recurrence post-transplantation is pervasive, DAAs have greatly improved SVR, and DAAs are better tolerated than interferon-based therapies, we anticipated that the use of DAAs would lead to a decline in the use of liver biopsies, which proved correct. In the era before the introduction of DAAs, the percentage of patients biopsied was greater than $50 \%$, compared to the era after the introduction of DAAs, when only slightly greater than $25 \%$ were biopsied. The data suggest that more patients are receiving preemptive treatment with DAAs instead of undergoing a liver biopsy to determine treatment. When examining patients in Era 2, 17 of the 67 patients had undetectable HCV RNA immediately preceding liver transplantation; thirteen patients had achieved SVR-12 prior to transplant, while four patients had undetectable HCV RNA, but had not achieved SVR-12. The achievement of HCV cure prior to transplantation in more than $20 \%$ of patients treated in Era 2 may be one reason why

Number of biopsies per year divided by number of liver transplant patients

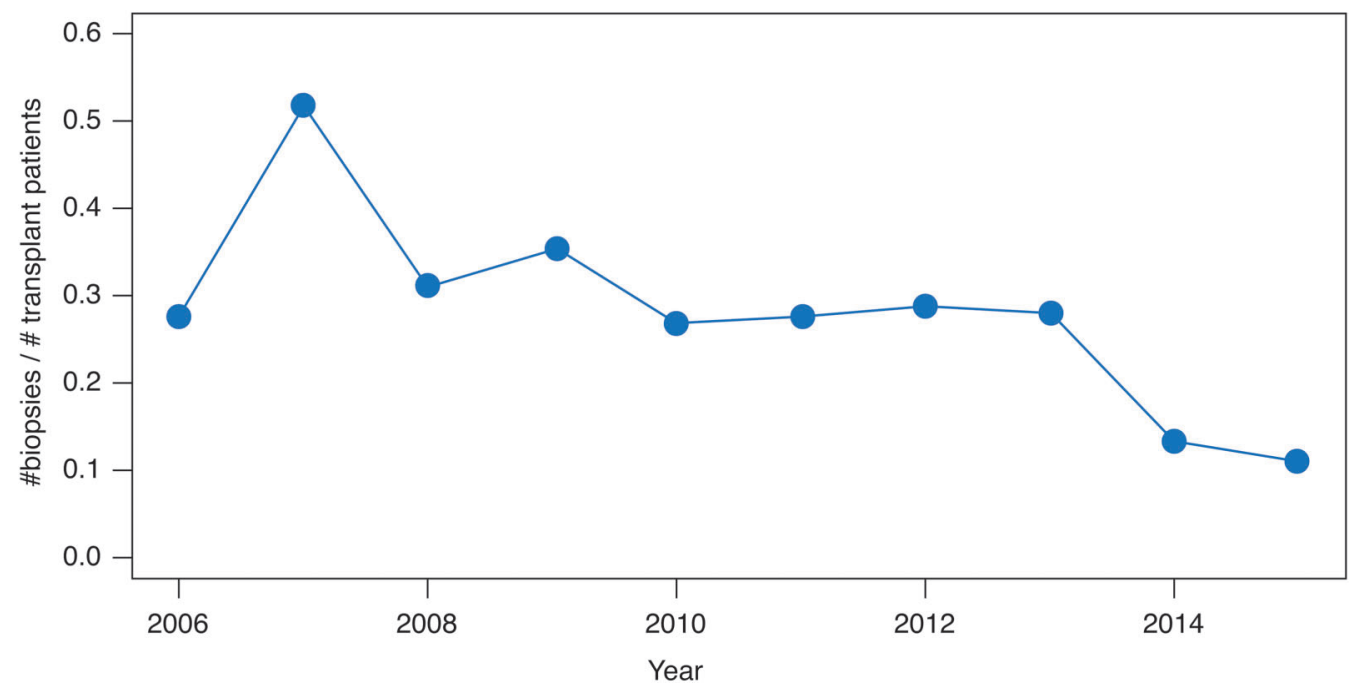

Fig. 2. Number of biopsies per year divided by the number of liver transplant patients from January 1, 2006 to October 1, 2015. 
Aby E. et al: Role of liver biopsy for HCV post-LT

Table 3. Cumulative percent of liver biopsies performed at 90, 180, 270 and 365 days post-transplantation separated by era, January 1, 2006 to October 1, 2015

\begin{tabular}{|c|c|c|c|c|c|}
\hline \multirow[b]{2}{*}{$\begin{array}{l}\text { Days from } \\
\text { transplant }\end{array}$} & \multicolumn{2}{|l|}{ Era 1} & \multicolumn{3}{|l|}{ Era 2} \\
\hline & $\begin{array}{l}\text { Cumulative number } \\
\text { of biopsies }\end{array}$ & $\begin{array}{l}\text { Cumulative percent } \\
\text { biopsied }\end{array}$ & $\begin{array}{l}\text { Cumulative number } \\
\text { of biopsies }\end{array}$ & $\begin{array}{l}\text { Cumulative percent } \\
\text { biopsied }\end{array}$ & $p$-value \\
\hline 90 & 69 & 17.1 & 11 & 16.4 & $>0.99$ \\
\hline 180 & 123 & 31.8 & 16 & 24 & $>0.99$ \\
\hline 270 & 148 & 39.6 & 16 & 24 & 0.138 \\
\hline 365 & 169 & 46.4 & 17 & 25.9 & 0.033 \\
\hline
\end{tabular}

patients in Era 2 were biopsied at a lower rate than patients in Era 1. In addition, in Era 1 almost $10 \%$ of patients were undergoing liver biopsy for HCV staging, while no patients in Era 2 underwent liver biopsy for HCV staging.

Despite the fact that fewer patients are undergoing liver biopsy post-transplantation, in certain circumstances liver biopsy continues to provide invaluable information necessary to guide patient care, including cases of suspected rejection. We posit that in the era of DAAs many of the patients undergoing liver biopsy within the first year post-liver transplantation are due to concern for rejection.

In addition to the introduction of DAAs, the management of HCV post-liver transplantation is changing with the integration of non-invasive measures of liver fibrosis into clinical practice. Moving forward, we may even see fewer liver biopsies because of the availability of these serum-based markers and imaging-based techniques. FibroSpect II (Prometheus Laboratories Inc.), a non-invasive diagnostic panel, is one such tool that is readily available at our institution. FibroSpect II, has been shown to successfully differentiate severe fibrosis (stages 3-4) from no to mild fibrosis (stages $0-1) .{ }^{12}$ While serum-based tests, such as FibroSpect II, are useful to establish either minimal fibrosis or cirrhosis, these modalities are less helpful with mid-range fibrosis or tracking progression of fibrosis. ${ }^{13}$ Although FibroSpect II is available for evaluation of fibrosis at UCLA, only 22 patients underwent
FibroSpect II during the study period, 2006 to 2015. When DAAs were approved, guidelines recommended HCV treatment regardless of liver disease severity; at UCLA, laboratory marker-based modalities, such as FibroSpect II, or imaging modalities, such as magnetic resonance imaging elastography, are typically only performed if there is a suspicion for cirrhosis.

Our study has a number of limitations that warrant discussion. First, it is a single center retrospective analysis, which limits generalizability given the geographic and institutional differences in liver transplantation. Its retrospective nature and the inherent limitations of an observational design may result in bias due to several confounding variables, including unmeasured patient characteristics. For approximately half of the patients in Era 1 and a quarter of the patients in Era 2, the genotype was unknown. Genotype is important in HCV management decisions, given that certain genotypes, including HCV $1 \mathrm{~b}$ and 4 , are known factors that predict a poor response to standard anti-viral therapy. ${ }^{14}$ The era after the introduction of DAAs has a small sample size, given the relatively recent development of non-interferon DAA regimens, and consequently some patients had only 6 months of follow up. However, it is important to recognize that the vast majority of initial acute rejection episodes occur within the first 6 months of transplantation. ${ }^{15-17}$

Time to first biopsy

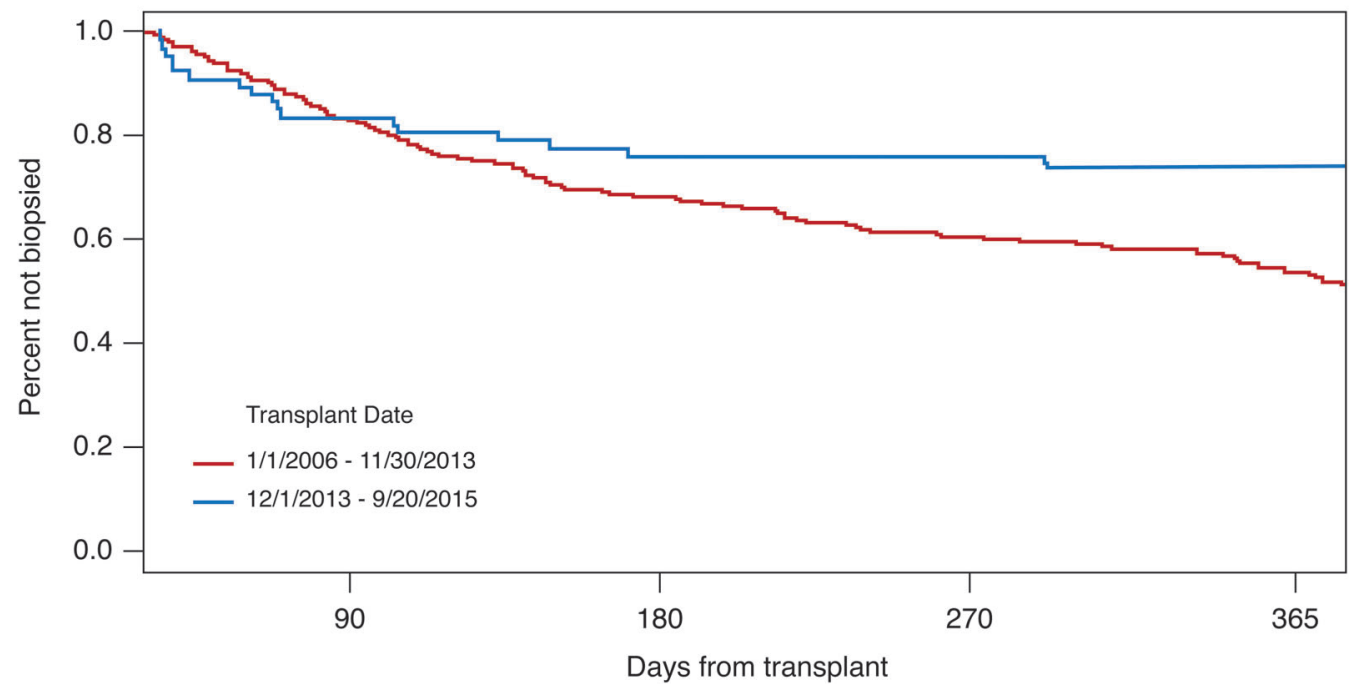

Fig. 3. Percentage of patients biopsied post-liver transplant for hepatitis C (HCV), separated by era ( $p=0.033$ at 365 days). 
The results of our single center study demonstrate that use of the combination of sofosbuvir and simeprevir is efficacious, safe and tolerable. This combination of DAAs should be considered in liver transplant recipients with recurrent HCV who are candidates for antiviral therapy. Further studies are needed compare the utility of sofosbuvir and simeprevir with other HCV non-interferon-based therapies.

The results of our study indicate that the introduction of DAAs is changing the role of liver biopsy post-liver transplantation. Given the substantially improved tolerability, SVR, and safety of DAAs against HCV, there is increasing interest in avoiding the potential complications of a liver biopsy. Thus, patients are more likely to be treated for HCV prior to transplantation and to be treated for HCV recurrence posttransplantation without a liver biopsy prior to initiation of therapy. Further studies are needed to determine if those who are biopsied less frequently have improved outcomes compared to those who are biopsied more frequently. Additional prospective studies are needed to develop algorithms for the use of liver biopsy in the evaluation and treatment of $\mathrm{HCV}$ recurrence post-liver transplantation and to understand which subgroups of patients with HCV recurrence posttransplantation may benefit from liver biopsy.

In summary, this study confirmed that increased use of DAAs has led to a decline in the use of liver biopsies and thus are changing the management of HCV liver disease post-liver transplantation.

\section{Conflict of interest}

The authors have no conflict of interests related to this publication.

\section{Author contributions}

Study concept and design (EA, MJ, SF, SS), acquisition of data $(E A, M J, S F)$, analysis and interpretation of data (EA, MJ, JG), drafting of the manuscript (EA, SS), critical revision of the manuscript for important intellectual content (EA, MJ, SF, RWB, SS), statistical analysis (JG), obtained funding (not applicable), study supervision (RWB, SS).

\section{References}

[1] Chak E, Talal AH, Sherman KE, Schiff ER, Saab S. Hepatitis C virus infection in USA: an estimate of true prevalence. Liver Int 2011;31:1090-1101. doi: $10.1111 / \mathrm{j} .1478-3231.2011 .02494 . x$
[2] Ly KN, Xing J, Klevens RM, Jiles RB, Ward JW, Holmberg SD. The increasing burden of mortality from viral hepatitis in the United States between 1999 and 2007. Ann Intern Med 2012;156:271-278. doi: 10.7326/0003-4819156-4-201202210-00004

[3] Kim WR, Lake JR, Smith JM, Skeans MA, Schladt DP, Edwards EB, et al. OPTN/SRTR 2013 Annual Data Report: liver. Am J Transplant 2015;15 Suppl 2:1-28. doi: 10.1111/ajt.13197.

[4] Forman LM, Lewis JD, Berlin JA, Feldman HI, Lucey MR. The association between hepatitis $C$ infection and survival after orthotopic liver transplantation. Gastroenterology 2002;122:889-896. doi: 10.1053/gast.2002.32418.

[5] Féray C, Caccamo L, Alexander G], Ducot B, Gugenheim J, Casanovas T, et al. European collaborative study on factors influencing outcome after liver transplantation for hepatitis C. European Concerted Action on Viral Hepatitis (EUROHEP) Group. Gastroenterology 1999;117:619-625. doi: 10.1016/ S0016-5085(99)70454-3.

[6] Sánchez CL, Len O, Gavalda J, Bilbao I, Castells L, Gelabert MA, et al. Liver biopsy-related infection in liver transplant recipients: A current matter of concern? Liver Transpl 2014;20:552-556. doi: 10.1002/lt.23817.

[7] Regev A, Berho M, Jeffers LJ, Milikowski C, Molina EG, Pyrsopoulos NT, et al. Sampling error and intraobserver variation in liver biopsy in patients with chronic HCV infection. Am J Gastroenterol 2002;97:2614-2618. doi: 10. 1111/j.1572-0241.2002.06038.x.

[8] Kohli A, Shaffer A, Sherman A, Kottilil S. Treatment of hepatitis C: a systematic review. JAMA 2014;312:631-640. doi: 10.1001/jama.2014.7085.

[9] Lam BP, Jeffers T, Younoszai Z, Fazel Y, Younossi ZM. The changing landscape of hepatitis $C$ virus therapy: focus on interferon-free treatment. Therap Adv Gastroenterol 2015;8:298-312. doi: 10.1177/1756283X15587481.

[10] Ermis F, Senocak Tasci E. New treatment strategies for hepatitis C infection. World J Hepatol 2015;7:2100-2109. doi: 10.4254/wjh.v7.i17.2100.

[11] Shiffman ML, James AM, Long AG, Alexander PC. Treatment of chronic $\mathrm{HCV}$ with sofosbuvir and simeprevir in patients with cirrhosis and contraindications to interferon and/or ribavirin. Am J Gastroenterol 2015;110: 1179-1185. doi: 10.1038/ajg.2015.218.

[12] Jeffers LJ, Cortes RA, Bejarano PA, Oh E, Regev A, Smith KM, et al. Prospective evaluation of FIBROSpect II for fibrosis detection in hepatitis $C$ and $B$ patients undergoing laparoscopic biopsy. Gastroenterol Hepatol (N Y) 2007; 3:367-376.

[13] Lackner C, Struber G, Liegl B, Leibl S, Ofner P, Bankuti C, et al. Comparison and validation of simple noninvasive tests for prediction of fibrosis in chronic hepatitis C. Hepatology 2005;41:1376-1382. doi: 10.1002/hep.20717.

[14] Roche B, Samuel D. Risk factors for hepatitis C recurrence after liver transplantation. J Viral Hepat 2007;14 Suppl 1:89-96. doi: 10.1111/j.13652893.2007.00920.x.

[15] Wiesner RH, Demetris AJ, Belle SH, Seaberg EC, Lake JR, Zetterman RK, et al. Acute hepatic allograft rejection: incidence, risk factors, and impact on outcome. Hepatology 1998;28:638-645. doi: 10.1002/hep.510280306.

[16] Wiesner RH, Ludwig J, Krom RA, Hay JE, van Hoek B. Hepatic allograft rejection: new developments in terminology, diagnosis, prevention, and treatment. Mayo Clin Proc 1993;68:69-79. doi: 10.1016/S0025-6196(12) 60022-6.

[17] Gómez-Manero N, Herrero JI, Quiroga J, Sangro B, Pardo F, Cienfuegos JA et al. Prognostic model for early acute rejection after liver transplantation. Liver Transpl 2001;7:246-254. doi: 10.1053/jlts.2001.22460. 